\title{
MASS-LUMINOSITY RATIOS AND SIZES OF GIANT ELLIPTICAL GALAXIES
}

\author{
IVAN R. KING \\ Berkeley Astronomy Dept. \\ and \\ RUDOLPH MINKOWSKI \\ Radio Astronomy Laboratory, University of California, Berkeley, Calif. U.S.A.
}

\begin{abstract}
Observations of velocity dispersion and of light distribution at the centers of 22 galaxies, mostly ellipticals give $M / L$ values of 15 to 40 for giants of all luminosities with smaller values for intrinsically faint galaxies. Core radii within which surface brightness drops by a factor of two are typically $50-100 \mathrm{pc}$ with a range from 2 to several hundred parsecs. Elliptical galaxies fit the modified isothermal models used for clusters, while later types have a flatter density gradient. Complete results will be published elsewhere.
\end{abstract}

Spectroscopic determination of the stellar velocity dispersion at the centers of giant galaxies makes it possible to determine central mass-luminosity ratios without recourse to the unverified assumptions implied by direct use of the virial theorem. (Those assumptions are that stars of all luminosities and in all locations have the same velocity dispersion and that the mass is distributed the same as the light.) The present technique involves a self-contained analysis of a small central region; essentially it compares an rms velocity with a scale height to find the force of gravity, which leads to a central mass density. In addition to the velocity dispersions the new data consist of photometry of the central peak of brightness. The remaining uncertainties come from the correction for atmospheric seeing and of course the uncertain Milky Way absorption and cosmic distance scale. (The results below assume $H=100 \mathrm{~km} \mathrm{~s}^{-1} \mathrm{Mpc}^{-1}$ and $A_{B, \text { pole }}=0^{m}$.3.)

Values of the central $M / L$ have been derived for 18 elliptical galaxies, $2 \mathrm{~S} 0$ 's, and $2 \mathrm{Sb}$ 's. The results lead to the following generalizations. (1) There is no clear difference in $M / L$ between the centers of ellipticals and the centers of S0's and Sb's. (2) Systems with integrated absolute magnitudes fainter than -19 have a distinctly lower central $M / L$. (3) Among the brighter systems there is no noticeable correlation of central $M / L$ with absolute magnitude. Values from 15 to 40 for giant systems probably represent a real range. (4) The $M / L$ values at the centers of giant galaxies are in good agreement with the results derived by Spinrad through photoelectric analysis of the spectra of a few systems.

The photometry was aimed at high resolution in the centers of the galaxies but was also extended far out into the envelopes. Its conclusions are as follows. (1) The central brightness peaks are small, with the surface brightness dropping by a factor of two from its central value typically in a 'core radius' of 50 to $100 \mathrm{pc}$ ( 1 to $2 \mathrm{~s}$ of arc at $10 \mathrm{Mpc}$ ). (2) Core radii range from $2 \mathrm{pc}$ for M31 and M32 to many hundreds of parsec for some of the Virgo giants. (3) Except for a scale factor, elliptical galaxies 
have remarkably similar profiles. They fit the modified isothermal models previously developed for star clusters; the envelopes are also very close to the laws suggested by Hubble and by de Vaucouleurs, but neither of those laws fits the cores. (4) Up to the point where the present study loses the elliptical galaxies in the sky noise, the galaxies show no clear limits. (5) A small amount of data on the later-type galaxies confirms their well-known flatter density gradient.

This project also includes studies of ellipticity profiles and rotations of several of the galaxies. These will be reported on at a later date.

\section{Discussion}

Woltjer: Could you give an estimate of the upper limit to the mass of a massive body at the center of your systems?

King: You can put a moderately massive object in the center, but you cannot use the high stellar velocity dispersion as an argument for its existence. My dynamical argument says that the velocity dispersion must be due to gravitating material that has the same extended distribution as the stars. An additional massive object is limited to a size that will not appreciably affect the gravitational field within one core radius, because the stars would then show an unmistakably different luminosity profile. I think that the practical upper limit to a central object is perhaps a tenth of the core mass.

Tifft: Do you have star/light distributions for galaxies with sharp centers, more sharp that is than NGC 4472 which is relatively diffuse?

King: Yes, I have some cases with sharper centers, where the central profile is nevertheless resolved. 